\title{
SIR JAMES LIGHTHILL
}

James Lighthill died on 17 July 1998, at the end of a ten-hour swim round the Channel Island of Sark. He had earlier, at age 49, been the first person ever to do this, and he was carrying out the swim for the seventh time when the exertion revealed a mitral valve weakness which had never been diagnosed, and which led to his sudden death in the water. The swim was one of many long 'adventure swims' which Lighthill liked to take, all characterized by strong tidal currents and often heavy seas. And Lighthill took much pleasure through exercising his comprehensive understanding of fluid mechanics first in preparing for them through study of local conditions and then in adapting his performance when, as often, he found that in practice the currents were not as charted and, in fact, often more treacherous.

Many obituary notices have already appeared in the national press in the UK and USA, and now in the newsletters and journals of learned societies; and extensive conspectuses of Lighthill's contributions to fluid mechanics and applied mathematics, and to science generally and to the administration of science, will be published in Annual Review of Fluid Mechanics (2000), and in Biographical Memoirs of Fellows of the Royal Society (2000). The reader will learn, from those accounts, of the unique range and depth of Lighthill's contributions; and virtually all readers should expect to be surprised and impressed to read of facets of Lighthill's work of which they were previously totally unaware.

This notice is written for readers of Journal of Fluid Mechanics now and in the future. Lighthill performed major service to the worldwide fluid mechanics community through JFM in at least five ways. First, he published in JFM a total of 26 papers, all but one under his sole authorship, the first in vol. 1, 1956, pp. 31-53, the most recent in vol. 239, 1992, pp. 551-606, and through these papers, among which are many of his most innovative, established the remit of JFM as encompassing, at the very least, topics as diverse as dissociating gases, swimming of slender fish, wave generation by wind, rotating fluids, physiological fluid dynamics, wave energy extraction devices, cochlear mechanics, offshore structure fluid loading, biofluiddynamics, and acoustic streaming. Amusingly, and characteristically, Lighthill holds the record for both the shortest and the longest titles of JFM papers ('Drift', vol. 1, 1956, pp. 31-53; and 'Biofluiddynamics of balistiform and gymnotiform locomotion. Part 2. The pressure distribution arising in two-dimensional irrotational flow from a general symmetrical motion of a flexible flat plate normal to itself', vol. 213, 1990, pp. 1-10, respectively).

Second, Lighthill was an Associate Editor of JFM from its inception in May 1956 to the end of June 1979, and listed this fact as an Honour in his curriculum vitae. We do not have complete statistics on the handling of JFM submissions for the first part of that period, but between January 1967 and June 1979, Lighthill dealt with 929 papers and accepted 515. The number of papers he handled exceeded that of any other Associate Editor, and was only marginally exceeded by those handled by Editors-in-Chief Batchelor and Moffatt. He dealt with papers, as one might expect, in a highly individual way, not shirking from accepting - or rejecting-a paper entirely on his own authority, nor indeed shirking from sending authors, on occasion, reports attributed to three anonymous referees A, B and C, each of whom was simply Lighthill himself. His acceptance ratio, 0.55 on the figures quoted above, was significantly higher than that of any other JFM Editor or Associate Editor, and 
consistently so for one 6-month reporting period after another. In large part-and the detailed files are confirmation-this stemmed from his wish to be encouraging, wherever possible, to scientists of promise - whether they were known to him or notand to scientists working in disadvantaged conditions. And his high acceptance ratio was not simply a reflection of well-intentioned acceptance of marginal or even weak papers, but rather a reflection of his willingness to put in much work with authors to improve papers which he thought contained interesting and significant results. His anomalously high acceptance ratio was - to the amusement of members of the JFM Editorial Team - the subject of repeated implicit criticism by our Founding Editor George Batchelor, who in his six-monthly newsletters to the Team invariably drew attention to the need for 'one's' acceptance ratio to lie within the range 0.43 to 0.47 . Lighthill of course registered the rebuke, and carried on as before; and Batchelor, knowing perfectly well how things would go, both appointed Lighthill from the start, and persuaded him to continue as hard-working Associate Editor for 24 years!

Third, while discharging his own editorial duties, Lighthill often acted as a referee for other JFM editors (and, of course, for the editors of many other journals). He could be harshly critical, and critical at considerable length - as I know to my cost!but the abiding memory which many will have had of Lighthill as a referee (there was never any trouble identifying him as such, even if protocol required him simply to be addressed as Referee A, or whatever) is of someone of remarkably broad and penetrating grasp, genuinely working with the author to make good work better.

Fourth, he was an eloquent reviewer of books. We have in JFM four particularly noteworthy reviews by Lighthill: of The Structure of Turbulent Shear Flow by A. A. Townsend (vol. 1, 1956, pp. 554-560); of Jets, Wakes and Cavities, by Garrett Birkhoff and E. H. Zarantonello (vol. 3, 1958, 437-440); of Collected Works of R. T. Jones (vol. 84, 1978, pp. 598-608); and of The Mechanics of the Circulation, by G. G. Caro, T. J. Pedley, R. C. Schroter and W. A. Seed (vol. 90, 1979, pp. 794-797). No one reading these can fail to be impressed, of course, with the breadth of knowledge that Lighthill brings to bear on major books in these four vastly different fields within fluid mechanics. More important, however, is the way that breadth of knowledge is deployed, first to establish a good feel in the mind of the general reader of JFM for the nature of the field and its place in relation to other fields, and then to probe various aspects of the book under review from many angles, ranging from the historical through the highly technical to the interpretative and expository, before concluding with a firm endorsement (at least in the cases mentioned) of the virtues of the book and its place in the scheme of things. Reviews of such scholarly depth would, of course, find a prominent place in the publication lists of the reviewer in some other subjects. Their importance is all the greater as subjects sub-divide and acquire their own specialist communities, conferences and even journals; and I hope that, through the good work of John Rallison as JFM Book Review Editor, JFM will be able to provide more reviews with the broad authority of those contributed by Lighthill.

Lighthill could be critical, certainly, but never aggressively so in the face of a serious attempt to address fluid mechanics topics in a substantial book. Listen to his admonition of his great mentor Sydney Goldstein in a review (vol. 11, 1961, pp. 319-320) of Lectures on Fluid Mechanics by S. Goldstein and J. M. Burgers: "One's only regret, in a book like this aimed at giving a first taste of fluid mechanics, is the paucity of figures (seventeen altogether). Even though it had been necessary to accept what it is not surprising to find the famous editor of Modern Developments in Fluid Mechanics calling 'one glaring and horrible omission - the omission of any comparison of theoretical with experimental results', nevertheless some diagrammatic 
representations of even calculated flow fields would have helped readers, unfamiliar with the subject's flavour, to appreciate that spicy component imparted to it by retinal reception of flow figuration.”(!)

And fifth, in his own writing, in his book reviewing, in his refereeing and in his editorial work, Lighthill always laid great stress on the importance of clear and attractive scientific writing, a matter on which he and George Batchelor were entirely at one, even if their own styles of writing were radically different. Lighthill credited the great 19 th century scientists for his concern for incisive and appropriately colourful exposition. "I love the 19th century writers. I call them writers because, although they were wonderful researchers, they wrote up their research so splendidly. Some of Stokes' papers are just an absolute joy to read, brilliant writing. Of course he shares this with Rayleigh..." (interview between Lighthill and Mr Robert Bruen, December 1992).

For JFM, as for so many other activities, Lighthill made major contributions, sustained over decades. The world community in fluid mechanics is greatly the richer for these contributions; and greatly the poorer for his loss.

D. G. CRighton 